\title{
MANAGEMENT OF ORTHODONTIC PATIENTS IN COVID-19 PANDEMIC, A SINGLE CENTER INITIAL EXPERIENCE
}

\author{
Aliaa A. Khadre* and Ahmed Elkady**
}

\begin{abstract}
The year 2020 will be marked for years to come with pandemics related to the new coronavirus disease 2019 (COVID-19) experiencing lockdowns, curfews and quarantine in a trial to create social distancing and reduce the transmission of the highly infective novel corona virus related to severe acute respiratory syndrome coronavirus-2 (SARS-CoV-2). It was considered as highly infective with a global concern of transmission as per world health organization (WHO). With the lockdown only emergency dental treatments were allowed with the complete closure of the orthodontic practice in a trial to help flatten the curve.
\end{abstract}

Aim: The aim of this clinical report is to describe the clinical outcome of orthodontist and assistant who took care of several COVID-19 cases possibly carriers of SARS-COV-2 or reported recent infection and recovery. The possible measures that can keep both dental health care workers (DHCWs) and patients safe added to the actions taken to help adapt to the new normal were elaborated.

Methods: A case series of one carrier and other five cases of recently recovered SARS-CoV-2 patients were managed in our orthodontic clinic. Different scenarios will be discussed for the purpose of comparing the management and highlighting the possible measures that were applied and precautions that could make the practice safer for both the orthodontist, assistant and patients following up in our clinic. Furthermore, we followed up other patients who had procedures in same clinic during upcoming 48 hours after each patient visit for 14 days through phone calls or recall visits.

Results: No transmission of infection to the dental health care workers as per nasopharyngeal swab. No transmission of infection as well for the follow up patients who had procedures in same clinic during upcoming 48 hours.

Conclusion: The infection control measures described in this clinical report were sufficient enough to prevent transmission of infection from a confirmed carrier patient.

KEYWORDS: COVID-19, Orthodontics, Health workers, Teledentistry, Aerosol procedures.

* Faculty of Dentistry, Orthodontic Department, Suez Canal University, Ismailia, Egypt.

** Mataria Teaching Hospital, Internal Medicine and Neurology Department, Cairo, Egypt. 


\section{INTRODUCTION}

The novel severe acute respiratory syndrome coronavirus-2 (SARS-CoV-2) that recently outbreak in Wuhan, China and has spread globally, was reported to be highly infectious compared to previous SARS-CoV and middle East respiratory syndrome coronavirus (MERS-CoV) ${ }^{[1]}$ Reports regarding spread of infection among the health care professionals and from patients to health care workers in china with $(1.1 \%)$ deaths, caused a global concern regarding infection control in medical facilities [2]. World health organization (WHO) strategic objectives were to interrupt human-to-human transmission via applying social distancing and wearing protective equipments ${ }^{[3]}$. As for dentistry and due to the aerosol generating procedures and the close proximity to the patients that makes social distancing extremely difficult if not impossible the risk was considered very high ${ }^{[4]}$.

With the flow of knowledge about the effectiveness of personal protective equipment (PPE) for dental health care workers and the lack of reports, dentists and orthodontists must comply with the most vigilant attitude towards infection control $^{[5]}$. Dental treatments, but emergencies, were prohibited by Ministry of Health $(\mathrm{MOH})^{[6]}$. Orthodontics is the branch of dentistry that nourishes and fleurs whenever life eases, complete shutdown of the practice discouraged new patients from seeking aesthetic treatments and put our follow up patients on hold. All we have seen in the clinic for months were broken lingual retainers impinging on the tongue and the patients suffering from trauma, ulcers or bleeding from poking archwires of broken fixed appliances to a lesser extent.

Caprioglio and colleagues ${ }^{[7]}$, provided detailed guidelines as to the management of emergencies in orthodontic office, they recommended training of the health care providers to the web-based communication systems. They reported WhatsApp, as the most popular and usable global mobile messenger application with 1.6 billion users on a monthly basis ${ }^{[8]}$. It can be used as the main and most common virtual communication tool between the clinician and the patient to follow up during emergencies throughout the lockdown, and in the new normal. This is very close to the concept of "Teledentistry" that had been used for long as an orthodontics consultation tool in deprived areas with limited access to proper services ${ }^{[8]}$, and for oral health education ${ }^{[9]}$. During the pandemics we enjoyed using the virtual online tool provided by the hospital for triage, patient categorization and dealing with moderate and high-risk patients.

After almost two months of complete closure and by the $15^{\text {th }}$ of May 2020, the MOH declared the reopening of the dental clinics to regular services exercising extreme caution and applying all infection control protocols considering patients and operators safety. Social distancing and personal hygiene were declared in all premises however, for the dental office extra measures and precautions must be implemented to overcome the increased risk of transmissibility through droplets and aerosol generating dental procedures. Orthodontics as a branch of dentistry is associated with both close proximity to the patients for average of 20-30 minutes and aerosol generation making it of high risk. British Orthodontic Society (BOS) ${ }^{[10]}$, classified orthodontic procedures to Aerosol generating procedures (AGPs) and non AGPs they provided detailed description of the PPE and clinical management, hinting on mask types and technique of properly donning and doffing PPE.

We returned to work in the new normal, in this report we described the clinical outcome of dental health care workers who took care of patients with SARS-CoV-2 during their routine follow up in our orthodontic clinic over one month from $15^{\text {th }}$ of May till $14^{\text {th }}$ of June 2020. Since reopening one of our patients was not known to be infected, while other cases were just recovered from recent infection. Furthermore, we followed up other patients who 
had procedures in the same clinic during upcoming 48 hours after each patient visit for 14 days.

\section{PATIENTS AND METHODS}

Following $\mathrm{MOH}$ recommendations after reopening, we started to use $\mathrm{MOH}$ check list score as a tool to categorize our patients risk for SARSCoV-2 infection (table 1) ${ }^{[6]}$, if the patient lived or worked in a place with COVID-19 positive cases in the last 14 days the risk is increased, moreover if present any of the clinical signs mentioned in the check list score, e.g. fever \& cough, the risk increases as well. It is of importance to imply the calculation of the risk score (table 1) either by the patient himself using online reservation tools or at the visual triage to classify the patients to: no risk, mild or moderate and sever risk ${ }^{[6]}$. Furthermore, we developed pathway flowchart (figure 1), to help directing every patient according to his clinical risk score and clinical condition. The possible infection control flow of events (figure1) were laid out in the light of the guidelines described by the $\mathrm{MOH}$ as per adequate management. And can be used in any orthodontic clinic for patients' categorization and screening. If the patient is of no risk (score of 0 ), management within the orthodontic clinic is appropriate for all types of procedures, however, if the score indicated moderate-severe risk patients $(\geq 4)$, elective orthodontic procedures should be delayed as for further assessment or online virtual consultation if appropriate, with referral to appropriate medical management with notification to the infection control department. Mild risk (score 1-3), will be referred to hospital emergency department for further assessment using chest imaging and blood investigations.

During one month after reopening, we followed up 90 patients. We identified six patients, one of them (Case 1) was pre-symptomatic, while others (Case 2-6), were just recovered from SARS-CoV-2 infection. We will highlight each scenario and our management protocol.
TABLE (1): Visual Triage Checklist for Acute Respiratory Infection approved by Ministry of Health (MOH):

\begin{tabular}{|c|c|}
\hline Risks for Acute Respiratory Illnesses & Score \\
\hline $\begin{array}{l}\text { A. Exposure Risks } \\
\text { 1. A contact with confirmed case of COVID-19 } \\
\text { in the last } 14 \text { days prior to symptoms onset. } \\
\text { OR } \\
\text { 2. Lived or worked in a facility known to be } \\
\text { experiencing an outbreak of COVID-19 in the } \\
\text { last } 14 \text { days prior to symptoms onset. }\end{array}$ & 3 \\
\hline $\begin{array}{l}\text { B. Clinical signs and symptoms } \\
\text { 1. Fever or recent history of fever. } \\
\text { 2. Cough (new or worsening). } \\
\text { 3. Shortness of breath (new or worsening). } \\
\text { 4. Headache, sore throat, or runny nose. } \\
\text { 5. Nausea, vomiting, and/or diarrhea } \\
\text { 6. Chronic renal failure, CAD/heart failure, Im- } \\
\text { munocompromised patient }\end{array}$ & $\begin{array}{l}4 \\
4 \\
4 \\
1 \\
1 \\
1 \\
1\end{array}$ \\
\hline Total Score* & \\
\hline
\end{tabular}

Scores: No risk=0, Mild risk =1-3,

Moderate risk $=4-6, \quad$ High risk $=\geq 7$

\section{Case 1:}

A 43 years old patient reported to the clinic on his scheduled monthly visit for fixed appliance adjustments and follow up. The patient was asymptomatic, his temperature was assessed at the visual triage and was 36.8 degrees Celsius and his risk score was zero (table 1), patient didn't report any risk factors and was fit for the procedure. According to our pathway (figure 1), arch-wires were changed in both arches and activation for space closure was done. The patient complained of excessive calculus deposits around his teeth in the lower anterior segment. Manual scaling for the affected area was done using sickle scaler, a total of 25 minutes' visit was rendered to the patient. During his procedure, both doctor and nurse were using personal protecting equipment's, PPE (N95 respirator, surgical mask, face shield and disposable surgical gowns). 


\section{Pathway for Orthodontic Patients in COVID-19 Pandemic}

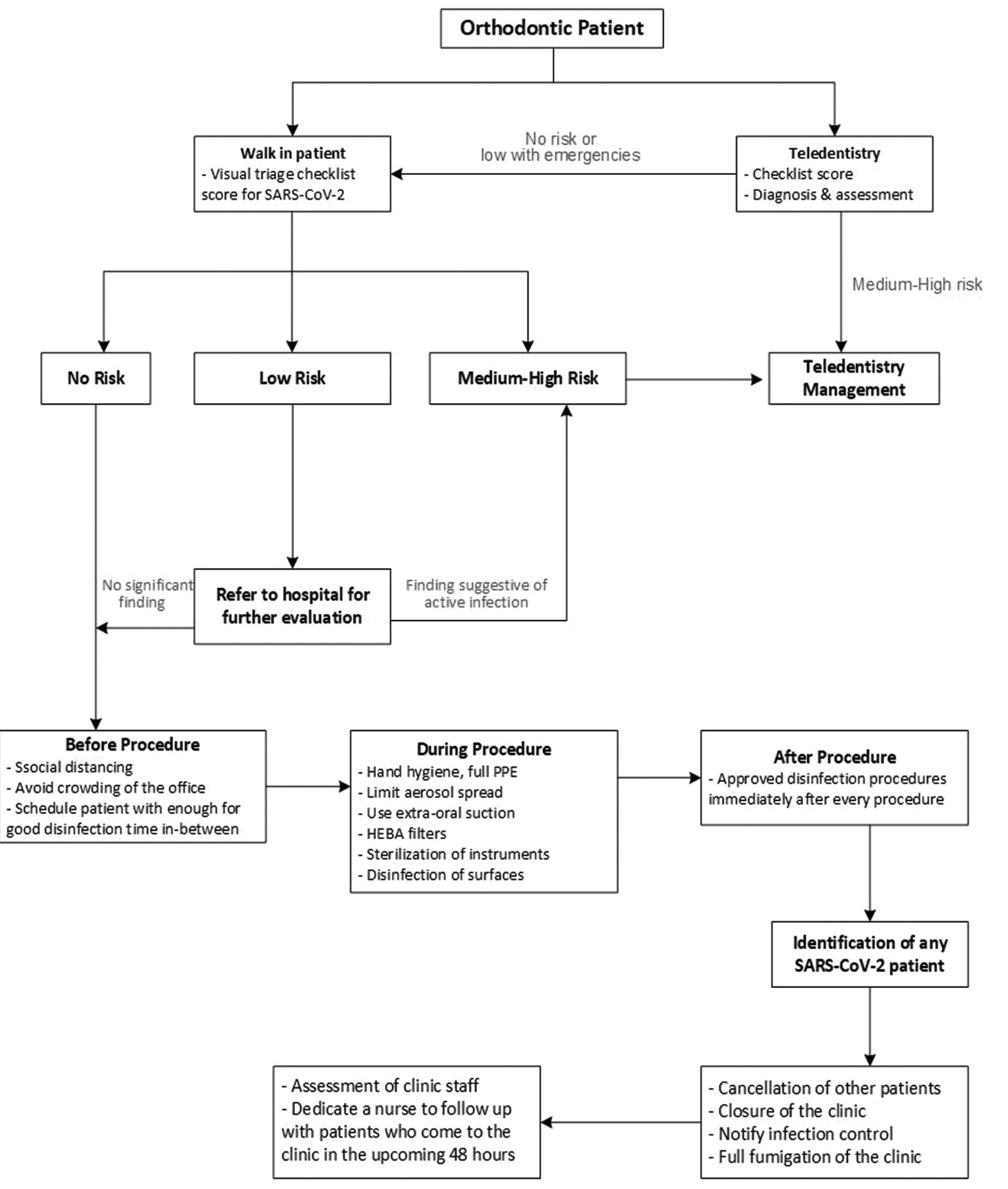

Fig. (1): flowchart demonstrating the possible flow events to manage orthodontic patients during COVID-19 pandemics. 
Later, 16 hours after the visit, the patient called the office and reported himself as a suspected COVID-19 patient he was suffering fever (38.5 degrees Celsius) and malaise, the patient was admitted to the emergency department. We immediately cancelled other patients follow up and infection control department was notified. Both orthodontist and nurse were self-isolated after nasopharyngeal swabs were taken, clinic and patients waiting areas were fumigated.

The patient suffered from sore throat, fever, cough, and shortness of breath. He was admitted for further investigations, a nasopharyngeal swab specimen was tested for SARS-CoV-2 and was positive. Computed topography (CT) chest showed typical bilateral ground glass appearance characteristic of SARS-CoV-2. The initial blood test results showed white blood cell count of, 4450/ $\mu \mathrm{L}$; with absolute lymphopenia of $0.85 / \mu \mathrm{L}$, serum ferritin, $347 \mathrm{ng} / \mathrm{mL}$.

However, all the staff test results were negative, with no symptoms related to SARS-Cov-2, therefore we started to follow up our patients again normally. We identified four patients who followed up in the same clinic in the following 2 days after reopening, hence they were followed up for 14 days for any symptoms via phone calls and/or recalling to the clinic.

\section{Cases 2-6}

During this month, another five asymptomatic patients who were recently recovered from SARSCoV-2 infection visited our clinic for their regular recall visits. They aged 23-43 years old, three were females, and four of them were health care workers. All five patient's symptoms were mild (fever, cough, and sore throat), and they were self-isolated at home with only symptomatic treatment. Their work up including laboratory and CT chest were within normal ranges, except mild lymphopenia in two patients. None of them were admitted to the hospital for any respiratory symptoms. After their second swab tested negative, they were assigned for their regular recall within 7-14 days. Changing archwires and activation of the appliances for space closure were the main features of all the cases.

Any patient who had procedures in same clinic during next 48 hours after any SARS-CoV-2 positive case, was followed up by dedicated nurse who interviewed them by phone calls. We identified 12 patients, 8 of them were males, their age ranged from (12-35) years. Using pre-prepared questioner, our nurse contacted all 12 patients and all of them did not report any symptoms related to SARS-CoV-2 infection within 14 days after their recall visit.

\section{DISCUSSION}

SARS-CoV-2 is here to stay and we need to develop a systematic flow of events that help maintain a safe working environment that generates the "New Normal" and implementing new gears to the orthodontic settings.

Health care workers (HCWs) were divided according to the risk of acquiring respiratory infectious disease to be either in high-risk department (HRD) with interventional medical or surgical procedures that generate respiratory aerosols, and other low-risk clinical departments regarded as general department groups. HRD group had 2.13 times higher risk in developing SARSCoV-2 (up to $13.53 \%$ in ICU) compared with the department groups group ${ }^{[11]}$.

Hence the orthodontic clinical setting could be considered HRD with increased risk for transmissibility, due its bioaerosol generation capacity with a toxic cloud spans from the floor to a height of six feet ${ }^{[12]}$. SARS-CoV-2 can stay viable for hours on metal and plastic surfaces with possible surface transmission ${ }^{[13]}$. A latest study provided evidence that SARS-CoV-2 widely distributed in the air with transmission distance might be up to 4 meters in hospital wards ${ }^{[14]}$. Hence, WHO 
recommended airborne precautions to medical staff ${ }^{[3]}$.

SARS-CoV-2 is more contagious than SARS$\mathrm{COV}$ and MERS-COV virus the middle east version ${ }^{[15]}$. WHO defined contact as: "Face-to-face" contact with a probable or confirmed case within 1 meter for more than 15 minutes"[16]. Orthodontic procedures involve close proximity to the patients, less than 1 meter for more than 15 minutes, which makes the operator and his assistance in a high chance of acquiring infectious diseases ${ }^{[15]}$. With several reports about spread of infection among health care workers, strict infection control procedure should be implemented to address the current situation.

Moreover, the incubation period for the virus reported a range from 5 days ${ }^{[17]}$, and up to 14 days $^{[18]}$, along which the patient can be asymptomatic yet, spreading infection in the dental office, unlike SARSCOV, COVID-19 is contagious during the latency period ${ }^{[19]}$. Building up SARS-CoV-2 herd immunity through natural infection is theoretically possible however, to the time being there is no solid evidence as such which means that a recovered COVID-19 patient (Case 2-6) may be still considered infectious till further reports are available ${ }^{[20]}$.

Recognizing the previously mentioned risk factors related to the new pandemics is our initial step to generate a safe flow of events in the clinic that will help reduce transmissibility and maintain the business running. The American Association of Orthodontists (AAO) task force interim recommended the adherence to social distancing and avoid crowding of the schedule and in the receptions, updated as to June $25^{\text {th }} 2020{ }^{\text {[21] }}$. In addition; self-protection by the use of correct PPE, identification and isolation of possible and known cases, are the main tools we have to overcome the current pandemic. Hand hygiene is considered the most important prevention measure for healthcareassociated infections.
Although, filters may be considerably less effective than other infection control measures, recently several orthodontic offices announced the introduction of HEBA filters to their offices. Can air purifiers help protect against infection? the answer is yes in theory ${ }^{[22]}$. Chen and colleagues have proven air purifiers can reduce the health-care workers exposure to aerosols and droplets in dental clinics significantly ${ }^{[22]}$. The efficiencies of HEPA filters are more than $95 \%$ for aerosols of diameter between 0.25 and $1.0 \mu \mathrm{m}$ and nearly $100 \%$ for those with diameter larger than $2.5 \mu \mathrm{m}$, COVID-19 is approximately 0.125 micron (125 nanometers) lying in the range of some HEPA filters ${ }^{[23]}$.

Refer to Caprioglio and colleagues ${ }^{[7]}$, orthodontic emergencies management during pandemics, classifying emergencies according to the type of the appliance considering the available virtual tools for management.

This crisis shed the light on the importance of increasing the use of teledentistry platforms for preliminary initial orthodontic diagnosis and consultations ${ }^{[8,25]}$. Teledentistry had been used mainly in dental education ${ }^{[9]}$, currently virtual online management is an indispensable tool in the new normal. Patients can have their initial assessment through sending their photographs both intraoral and/or extra oral to the orthodontist and having virtual online visit were the orthodontist can discuss with the patients their concerns about their smiles and the possible treatment options in addition to the estimated cost and possible outcomes. Some of the clinics even provide a frequently asked questions (FAQs) section for patients to give them a better acceptance and introduction to the new normal

Furthermore, patients with fixed orthodontic appliances should have close follow up using virtual online meetings that can help reassure and address their concerns about their tooth movements and reduce emergencies. 


\section{LIMITATIONS}

We recognize the limitations of this case series and acknowledge that additional studies are necessary to determine the best route to protect orthodontists from becoming infected with SARS$\mathrm{CoV}-2$ while they are providing care for patients within COVID-19 pandemics.

\section{CONCLUSION}

Through the previous few weeks, we witnessed several cases of SARS-CoV-2 recoverees and one unknown carrier confirmed later as SARS-CoV-2 positive. Using $\mathrm{MOH}$ risk score, our developed flowchart pathway and other infection control measures were up to the challenge and prove their effectiveness for protection of DHCW from infection spread through high risk field; hence, the office kept running daily without significant interruption to the time being.

\section{ABBREVIATIONS}

COVID-19: coronavirus disease 2019; SARSCoV-2: severe acute respiratory syndrome coronavirus-2; WHO: world health organization; MERS-CoV: middle East respiratory syndrome coronavirus; PPE: personal protective equipment; MOH: Ministry of Health; BOS: British Orthodontic Society; AGPs: Aerosol generating procedures; CT: Computed topography; HCWs: Health care workers; HRD: high-risk department; AAO: American Association of Orthodontists; FAQs: frequently asked questions.

\section{REFERENCES}

1. Xie M, Chen Q. Insight into 2019 novel coronavirus - An updated interim review and lessons from SARS-CoV and MERS-CoV. Int J Infect Dis. 2020;94:119-24.

2. Wang J, Zhou M, Liu F. Reasons for healthcare workers becoming infected with novel coronavirus disease 2019 (COVID-19) in China. J Hosp Infect. 2020;105(1):100-1.

3. WHO Recommendation for Infection prevention and control during health care when coronavirus disease (COVID-19) is suspected or confirmed. https://www.who.
int/publications/i/item/WHO-2019-nCoV-IPC-2020.4. Accessed 5 July 2020.

4. Spagnuolo G, De Vito D, Rengo S, Tatullo M. COVID-19 Outbreak: An Overview on Dentistry. Int J Environ Res Public Health. 2020;17(6):E2094.

5. Ferioli M, Cisternino C, Leo V, Pisani L, Palange P, Nava S. Protecting healthcare workers from SARSCoV-2 infection: practical indications. Eur Respir Rev. 2020;29(155):200068.

6. CDC/MOH guidance in COVID-19 (V. 1.3). https://www. moh.gov.sa/en/Ministry/MediaCenter/Publications/Pages/ covid19.aspx. Accessed 5 July 2020.

7. Caprioglio A, Pizzetti GB, Zecca PA, Fastuca R, Maino G, Nanda R. Management of orthodontic emergencies during 2019-NCOV. Prog Orthod. 2020;21(1): 10.

8. Stephens C, Cook J, Mullings C. Orthodontic referrals via TeleDent Southwest. Dent Clin North Am. 2002;46(3): 507-20.

9. Chen JW, Hobdell MH, Dunn K, Johnson KA, Zhang J. Teledentistry and its use in dental education. J Am Dent Assoc. 2003;134(3):342-6.

10. COVID19 BOS Advice I Recovery Phase Advice I What is appropriate orthodontic PPE https://www.bos.org.uk/ COVID19-BOS-Advice/Recovery-Phase-Advice/Whatis-appropriate-orthodontic-PPE . version 1.0 published May 2020 .Accessed July $5^{\text {th }} 2020$.

11. Ran L, Chen X, Wang Y, Wu W, Zhang L, Tan X. Risk Factors of Healthcare Workers with Corona Virus Disease 2019: A Retrospective Cohort Study in a Designated Hospital of Wuhan in China. Clin Infect Dis. [published online: March 17, 2020].

12. Malamed SF. Medical emergencies in the dental surgery. Part 1: Preparation of the office and basic management. J Ir Dent Assoc. 2015;61(6):302-8.

13. van Doremalen N, Bushmaker T, Morris DH, Holbrook MG, Gamble A, Williamson BN, et al. Aerosol and Surface Stability of SARS-CoV-2 as Compared with SARS-CoV-1 . N Engl J Med. 2020;382(16):1564-7.

14. Guo ZD, Wang ZY,Zhang SF, Li X, Li L, Li C, et al.Aerosol and Surface Distribution of Severe Acute Respiratory Syndrome Coronavirus 2 in Hospital Wards, Wuhan, China, 2020. Emerging Infect Dis. 2020;26(7):1583-91. 
15. Peng X, Xu X, Li Y, Cheng L, Zhou X, Ren B. Transmission routes of 2019-nCoV and controls in dental practice. Int J Oral Sci. 2020;12(1):9.

16. WHO Coronavirus disease (COVID-2019) situation reports. https://www.who.int/emergencies/diseases/novelcoronavirus-2019/situation-reports. Accessed 5 July 2020.

17. Lauer SA, Grantz KH, Bi Q, Jones FK, Zheng Q, Meredith HR, et al. The Incubation Period of Coronavirus Disease 2019 (COVID-19) From Publicly Reported Confirmed Cases: Estimation and Application. Ann Intern Med. 2020; 172(9):577-82.

18. Zhou P, Yang XL, Wang XG, Hu B, Zhang L, Zhang W, et al. A pneumonia outbreak associated with a new coronavirus of probable bat origin. Nature. 2020;579(7798):270-3 .

19. Liu Y, Ning Z, Chen Y, Guo M, Liu Y, Gali NK, et al. Aerodynamic analysis of SARS-CoV-2 in two Wuhan hospitals. Nature. 2020;582(7813):557-60.

20. Randolph HE, Barreiro LB. Herd Immunity: Understanding
COVID-19. Immunity. 2020;52(5):737-41. DOI:10.1016/j. immuni.2020.04.012.

21. AAO COVID-19 Resources for Orthodontists. https:// www1 .aaoinfo.org/covid-19/. Accessed 5 July 2020.

22. Chen C, Zhao B, Cui W, Dong L, An N, Ouyang X. The effectiveness of an air cleaner in controlling droplet/aerosol particle dispersion emitted from a patient's mouth in the indoor environment of dental clinics. J R Soc Interface. 2010;7(48):1105-18.

23. Chen C, Ji W, Zhao B. Size-dependent efficiencies of ultrafine particle removal of various filter media. Build Environ. 2019;160:106171.

24. Ng K, Poon BH, Kiat Puar TH, Shan Quah JL, Loh WJ, Wong YJ, et al. COVID-19 and the Risk to Health Care Workers: A Case Report. Ann Intern Med. 2020; 172(11):766-7.

25. Berndt J, Leone P, King G. Using teledentistry to provide interceptive orthodontic services to disadvantaged children. Am J Orthod Dentofacial Orthop. 2008;134(5):700-6. 